\title{
A load dependent metric for balancing Internet traffic in Wireless Mesh Networks
}

\author{
Hervé Aïache, Laure Lebrun, Vania Conan, Stéphane Rousseau \\ Thales Communications, 160 boulevard de Valmy \\ -BP82 -92704 Colombes Cedex -France \\ surname.name@fr.thalesgroup.com
}

\begin{abstract}
In Wireless Mesh Networks (WMNs) traffic is routed from mobile clients through a multihop wireless backbone to and from Internet Gateways (IGWs). Because of their limited number, IGWs become the major traffic bottlenecks. The purpose of this work is to explore the benefits of introducing load-dependent routing metrics to increase WMN capacity and performance. We use weighted shortest path routing and introduce LAETT a weight metric that captures both traffic load and link quality. We compare the scheme to ETT and MIC, two load independent metrics, and show in simulation its benefits for various network and traffic configurations.
\end{abstract}

\section{Introduction}

Mesh networks are wireless broadband networks composed of fixed Mesh Routers (MRs) and fixed or mobile wireless clients [1]. The benefits of WMNs include their low investment costs, ease of installation and incremental deployment. They provide viable alternatives to xDSL or cable solutions in urban areas. Because they are primarily used to provide Internet access, congestion builds up around the Internet Gateways (IGWs) imposing severe limitations to the achievable capacity of the whole WMN [2].

To provide maximum usage of the infrastructure or to maximize the capacity of the WMN $[\mathbf{1 2 , 1 3}]$, one tries to balance the load across the network. Past work has focused on topological load-independent weights (hop-count, raw link capacity, ...): ETX (Expected Transmission Count) exploits the total number of transmissions needed to transmit a packet based on packet loss in both directions [3], ETT (Expected Transmission Time) and WCETT (Weighted Cumulative Expected Transmission Time) weigh this measure by the size of the packet times the data rate [5]. Inter-flow and intra-flow interference estimates are also used to route traffic so as to limit interferences: MIC [4] combines the two independently and CATT [6] captures both simultaneously.
In this paper we further introduce an estimate of the remaining capacity on the link as a load-sensitive parameter in the metric to adapt better to actual available resources in the network. To avoid congestion one wishes to maximize the minimum remaining capacity of nodes in the network. In wired networks the inverse of the remaining capacity of the node can be used directly as the metric and this can be extended to the interference-prone wireless case [7]. This led us to define a new metric which combines the estimation of traffic load and link quality.

The load dependent metric is used with a weighted Dijsktra load balancing scheme. All packets from the same flow follow the same route; load balancing is achieved on a per flow basis and not at individual packet level avoiding much of the intricacies involved in packet reassembly and instabilities of routing decisions [8]. To evaluate the performances of the scheme we first consider a typical case of a multigateway WMN with a given traffic matrix. We compare the scheme to ETT and MIC, two load independent ones and demonstrate its performance benefits for different values of the number of gateways and its robustness in the presence of intra-mesh traffic.

The remainder of the paper is organized as follows. Section 2 presents the load aware routing protocol and the new metric that we introduce. Section 3 evaluates the scheme on a 4 gateway wireless mesh of 100 nodes. Section 4 discusses the influence of various parameters on the scheme. Section 5 concludes with future perspectives of work.

\section{Load aware routing}

The most critical element in our proposed routing scheme is the appropriate design of a metric that gives each link on the route a weight. The weights, once aggregated, discourage the routes going through already heavily loaded regions. Because they are traversing a wireless mesh, the routes should also limit the induced interferences, to preserve the overall capacity of the network. 


\subsection{Link metric}

LAETT, the metric that we propose, combines wireless access characteristics and load estimates. It consists in an adaptation of the ETT metric [5].

For link (i,j) between nodes $\mathbf{i}$ and $\mathbf{j}$, the Expected Transmission Time $E T T_{i j}$ is given by:

$$
E_{i j}=\operatorname{ETX}_{i j} \frac{S}{B_{i j}}
$$

where ETX $_{\mathrm{ij}}$ is the expected transmission count on link (i,j), $\mathbf{S}$ is the packet size and $\mathbf{B}_{\mathbf{i j}}$ is the effective bitrate (in packets per unit time). If $\mathbf{B}_{\mathbf{i}}$ is the transmission rate of node $\mathrm{i}, B_{i j}=\frac{B_{i}}{\gamma_{i j}}$, where $\gamma_{\mathrm{ij}}$ is the link quality factor (such as given by Table 2); the highest data rate is achieved for $\boldsymbol{\gamma}_{\mathbf{i j}}$ close to 1 when the link is of good transmission quality. When transmission quality degrades, $\boldsymbol{\gamma}_{\mathbf{i j}}$ increases and the $\mathbf{B}_{\mathbf{i j}}$ bitrate decreases.

To take into account the load, we introduce $R C_{i}$ the Remaining Capacity on each node, given by:

$$
R C_{i}=B_{i}-\sum_{k=1}^{k=N_{i}} f_{i k} \gamma_{i k}
$$

where the $f_{i k}$ are the transmission rates of the $N_{i}$ current flows that traverse node $i$. Note that the cost of a flow on the remaining capacity is weighted by the incurred PHY/MAC factor $\gamma_{i k}$ : good quality transmissions use less resources than bad quality ones.

The LAETT (Load Aware ETT) metric introduces the marginal cost of adding the new flow on the link for both its endpoints $i$ and $j$ : it measures the expected transmission time it would take to transmit the flow if it is allowed to use only the remaining capacity at both end points. We thus define $L A E T T_{i j}$ by:

$$
L O A D_{i j}=E T X_{i j} \times \frac{S}{\left(\frac{R C_{i}+R C_{j}}{2 \gamma_{i j}}\right)}
$$

ETX $_{i j}$ measures the number of MAC transmissions required to transmit successfully a message on the $(i, j)$ link. The second factor captures the remaining capacity at both end nodes. When two paths have the same cumulated weight in terms of ETX, the scheme favors the one with the most remaining capacity. Moreover, if all nodes have the same initial capacity $B$ and the network is unloaded, the LAETT metric is equal to the ETT metric.

\subsection{Routing protocol}

From a routing protocol point of view we consider that, due to its static nature, the mesh network runs a proactive link state protocol such as OLSR. Topology update messages are broadcast on a regular basis or triggered by topology changes. Routes are then computed by each node in a distributed fashion, using a weighted Dijkstra algorithm, the weights being given by Formula (3). The aggregate route cost is obtained as the sum of the non-negative LAETT weights on each link, so the routing metric is strictly isotonic and generates loop-free routes [10].

The actual implementation of the protocol on a real platform also requires the monitoring of the traffic on each MR to compute the link metric in Formula (3). The ETX value can be obtained by each node from its neighbors by exchanging periodic layer 3 hello messages on the broadcast channel [3]. A more precise value that takes into account the actual sizes of exchanged packets can be implemented with information from layer 2 [11]. The packet-pair algorithm can be used to estimate the available bandwidth on a link, thus providing $\boldsymbol{\gamma}_{\mathrm{ij}}$. It may also be obtained from the air interface. The remaining capacity computation further requires knowledge of the link quality, available bandwidth on the link (provided by packet-pair) which can be obtained from the air interface through the sending/receiving bitrates. For air interfaces that use the Demand Assignment Multiple Access-Time Division Multiple Access (DAMA-TDMA) technique such as WiMAX [15], the remaining capacity can be obtained from layer 2 in terms of free slots and completed by information on the used modulation schemes.

\section{Evaluation on Internet traffic}

In this section, we propose to highlight the gain one can expect by introducing load-dependent information in the routing decisions both in terms of network capacity and load distribution. We simulate a WMN and compare the performances of the LAETT metric to two load-independent state of the art metrics, ETT and MIC $[4,5]$.

\subsection{Set-up description}

We consider a Wireless Mesh Network spreading over an $800 * 600$ area. It is composed of 96 Mesh 
Routers (MRs) and 4 Internet Gateways (IGWs). All MRs and IGWs have wireless interfaces. The IGWs have an additional wired interface towards the Internet. The 4 IGWs (IGW0 through IGW3) are located on an even grid over the area. The corresponding network is shown in Figure 1 where IGW0 is top left, IGW1 bottom right, IGW2 bottom left and IGW3 top right. We assume that wired interfaces towards the Internet are not bandwidth limited.

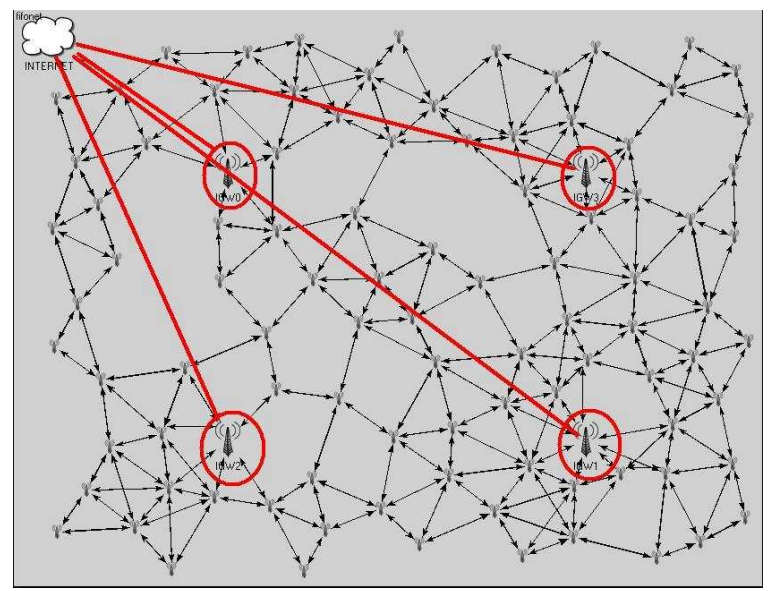

Figure 1 Example of a Wireless Mesh Network with 4 Internet Gateways and 96 Mesh Routers.

\subsection{Comparison assumptions}

At the PHY layer, we assume that the Signal to Noise Ratio (SNR) at the receiver falls off with D, the distance between the source and the destination according to a power law at a rate termed as path loss exponent $\boldsymbol{\alpha}$. Considering a peri-urban environment, we use $\alpha=3$, so SNR is proportional to $1 / D^{\alpha}$.

To fulfill the expected quality of service, the transmission data rate decays with the distance as shown in Table 2. The table gives the corresponding physical rates according to some distance threshold between each pair of nodes. These figures are based on results presented in [10].

\begin{tabular}{|l|c|c|c|c|c|}
\hline Distance $(\mathbf{m})$ & $\mathbf{> 1 6 0 0}$ & $\mathbf{> 8 0 0}$ & $\mathbf{> 5 6 0}$ & $\mathbf{> 4 8 0}$ & $\mathbf{> 4 0 0}$ \\
\hline Link factor $\boldsymbol{\gamma}$ & $\infty$ & 4 & 3 & 2 & 1 \\
\hline $\begin{array}{l}\text { Transmission } \\
\text { data rate } \\
\text { Mbps) }\end{array}$ & $\begin{array}{c}\text { Carrier } \\
\text { sense }\end{array}$ & 2 & 2,5 & 4 & 8 \\
\hline
\end{tabular}

Table 2 : Link quality factor and corresponding data rate according to the distance

We consider that appropriate network planning ensures that two neighboring nodes using the same waveform are distant enough to reduce interflow interference to a minimum.

In the simulations we adopt the MAC layer simplifications of [2]. MAC layer scheduling is omnipotent and achieves maximum concurrent transmissions among all nodes. This simplification does not impact the complexity of the bandwidth constrained routing problem which still remains hard [14]. It delivers upper bounds for the expected capacity gains and provides insights on the relative performances of the compared routing schemes.

Finally, we consider that the data rate requests of the flows is granted fairly by the transport protocol.

\subsection{Evaluation criteria}

Two measures are used to evaluate the performances of the routing decisions made by the ETT, MIC or LAETT metrics.

The first measure is the network capacity which is an estimate of the maximum amount of traffic that the network can support. The measurement proceeds as follows: a finite set of flows (origin destination pairs) is generated and the data rate of these flows is increased until the first node in the network is saturated (its remaining capacity in Formula (2) would go below 0 ).

The second measure monitors the load distribution among the nodes in the network. Congestion areas much depend on routing decisions. Proper distribution of traffic is a way to anticipate the possible saturation of some nodes when the load increases.

\subsection{Results}

The three metrics are compared by running simulations in the same conditions and for the same traffic patterns. In this Section we focus on Internet traffic only, as this is the main traffic type in a mesh network. The influence of intra-mesh traffic is discussed in Section 4.2.

We generate 450 Internet-WMN flows (flows between a MR in the WMN and the Internet). The MR of each flow is randomly chosen among the WMN MRs. All data rates are progressively increased until at least one of the MRs or IGWs runs out of resources. The results are averages over 200 runs.

\subsubsection{Network capacity}

In the case of Internet-WMN flows, the maximum capacity of the network is bounded by the cumulated available bandwidth of the 4 IGWs. As each IGW can 
receive at most $8 \mathrm{Mb} / \mathrm{s}$, and as there are $4 \mathrm{IGWs}$, an upper bound for the maximum capacity of the network is equal to $32 \mathrm{Mb} / \mathrm{s}$.

With the ETT and MIC metrics, the network capacity is $13.9 \mathrm{Mb} / \mathrm{s}$ (the flows have a bitrate of $31 \mathrm{~kb} / \mathrm{s})$. On the other hand, with the LAETT metric the network capacity reaches $19.8 \mathrm{Mb} / \mathrm{s}$ (the flows have a bitrate of $44 \mathrm{~kb} / \mathrm{s})$. This means a $42 \%$ increase in capacity over ETT and MIC and the capacity-ratio (over the maximum achievable $32 \mathrm{Mb} / \mathrm{s}$ ) is equal to $61.8 \%$.

This increased performance can be understood by looking at the balance of loads at and around the IGWs: using the load independent ETT and MIC metric, one of the 4 IGWs (IGW 0) supports more flows than the three other IGWs (see Figure 2). With the load dependent LAETT metric some of the flows are routed to the other IGWs in order to balance the load over the $4 \mathrm{IGWs}$; see Section 4.2 for further discussion of this phenomenon.

\subsubsection{Load distribution}

Let's compare the load distribution in the network for a cumulated data rate equal to $13.9 \mathrm{Mb} / \mathrm{s}$ (the maximum network capacity with ETT or MIC).

The average load of nodes (MRs and IGWs) is about $18 \%$ using either the ETT, MIC or LAETT metric. Some MRs are not loaded (less than 1\%): as one can anticipate, the nodes far from the IGWs are not used for relaying Internet traffic.

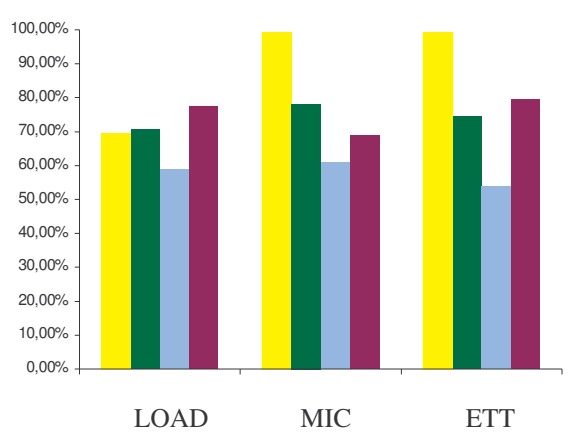

Figure 2: Comparison of the 4 hot spots (from left to right: IGW0, IGW1, IGW2, IGW3)

Conversely the most loaded nodes are located around the IGWs but the three metrics do not behave in the same way. Figure 2 compares the four hot-spot values (the maximum load around the four gateways) for the three metrics. With ETT and MIC congestion is located at IGW0 whereas the other areas are not over- loaded. With LAETT the load is distributed more evenly among the 4 IGWs. Furthermore Figure 2 shows that the hot-spots created by LAETT induce a maximum load of only $78 \%$ of the maximum load generated by ETT and MIC (100\% by construction).

In Figure 3, Figure 4 and Figure 5, the load distribution is shown for respectively ETT, MIC and LAETT. On the left, we present the hot spots location (in black) over the network area; on the right, we focus on the nodes remaining available bandwidth.

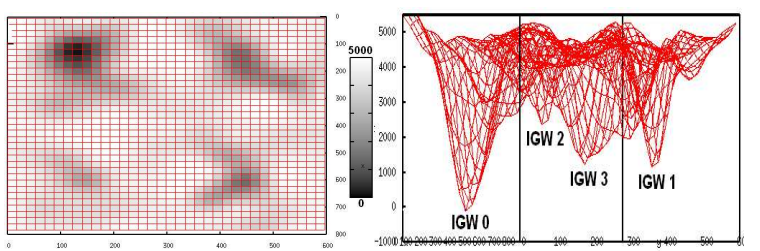

Figure 3: Load distribution using ETT

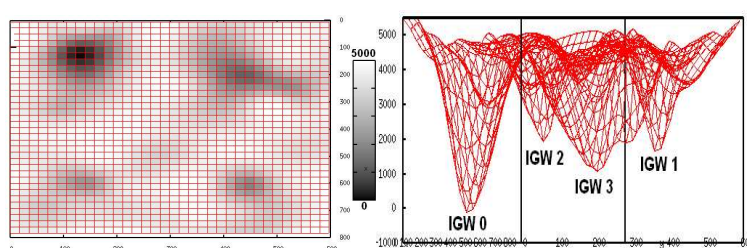

Figure 4: Load distribution using MIC

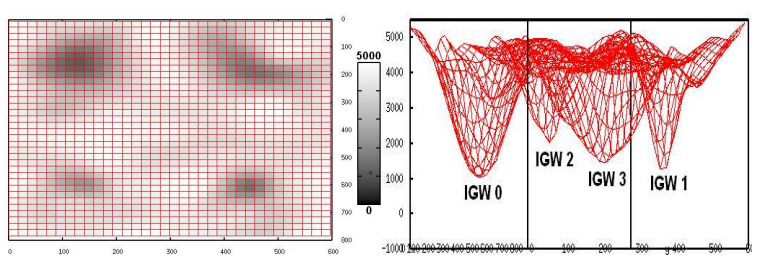

Figure 5: Load distribution using LAETT

According to simulation results, some flows that are routed to Internet via IGW 0 using the ETT and MIC metrics are routed to Internet via another IGW using the LAETT metric.

Thus, the LAETT metric gives better performance in terms of load balancing and resource management. Indeed the load lies between $60 \%$ and $80 \%$ for LAETT and between $60 \%$ and $100 \%$ for ETT and MIC. With LAETT routing decisions are made based on the current load status of the network, and some bottlenecks can be avoided by routing some traffic through other IGWs.

\section{Discussion and Analysis}

In this section, we discuss the influence of two parameters on the LAETT performances: the number of IGWs in the network and the presence of additional 
intra-mesh traffic (flows whose source and destination MRs are both in the WMN).

\subsection{Number of Internet Gateways}

We consider the same WMN as in Section 3 but we vary the number of IGWs from 1 to 4 . All traffic flows are Internet-WMN flows. We compare the network capacity for each case using the ETT, MIC or LAETT metric. Results are given in Table 3.

When the WMN is composed of only one IGW, the three metrics achieve the same capacity of $4.9 \mathrm{Mb} / \mathrm{s}$, to be compared to a theoretical maximum network capacity of $8 \mathrm{Mb} / \mathrm{s}$. The IGW is the bottleneck of the network and there is not enough path diversity for improving the routing decisions.

\begin{tabular}{|l|l|l|l|}
\cline { 2 - 4 } \multicolumn{1}{c|}{} & ETT & MIC & LAETT \\
\hline 1 IGW & $4.9 \mathrm{Mb} / \mathrm{s}$ & $4.9 \mathrm{Mb} / \mathrm{s}$ & $4.9 \mathrm{Mb} /$ \\
\hline 2 IGWs & $7.2 \mathrm{Mb} / \mathrm{s}$ & $6.7 \mathrm{Mb} / \mathrm{s}$ & $9.9 \mathrm{Mb} / \mathrm{s}$ \\
\hline 3 IGWs & $11.2 \mathrm{Mb} / \mathrm{s}$ & $9.9 \mathrm{Mb} / \mathrm{s}$ & $14.8 \mathrm{Mb} / \mathrm{s}$ \\
\hline 4 IGWs & $13.9 \mathrm{Mb} / \mathrm{s}$ & $13.9 \mathrm{Mb} / \mathrm{s}$ & $19.8 \mathrm{Mb} / \mathrm{s}$ \\
\hline
\end{tabular}

Table 3: capacity of the network

Adding IGWs to the WMN generates shorter routes, so it increases overall performances; but it also gives more possibilities for choosing through which IGW to route traffic. As can be seen from Table 3, LAETT takes better advantage of this increased flexibility than ETT or MIC. All three cases of a number of IGWS of 2, 3 and 4, behave in a similar way: for ETT and MIC, IGW0 is saturated first and the three IGWs are at most $80 \%$ loaded and LAETT balances the load more evenly on the IGWs.

Wireless Mesh Networks tend to have more and more MRs and IGWs in order to cover large areas. In this context, communications between the WMN and Internet will be possible using different IGWs. This work shows the benefits one can expect from using a load dependent metric to make more informed routing decisions, balance the load and avoid bottlenecks.

\subsection{Intra-mesh traffic}

The majority of mesh traffic is with the Internet. But there can still be some local intra-mesh traffic (for local phone calls for example). In this section we evaluate the impact of this traffic on the performance of our proposed scheme.

Let's come back to the network with the 4 IGWs and introduce $50 \%$ of intra-mesh traffic (the other $50 \%$ remaining Internet-WMN traffic). For both intra-mesh traffic and Internet-WMN flows sources are chosen at random. For intra-mesh traffic, the destination is randomly chosen among the MRs of the WMN.

We generate 450 flows and increase the data rate of each flow until the maximum network capacity. The study is based on 200 simulation runs. Table 4 shows the minimum network capacity, the maximum network capacity and the average network capacity obtained for all runs.

Using ETT and MIC metrics, the average network capacity is about $10 \mathrm{MB} / \mathrm{s}$. The minimum network capacity is about $9 \mathrm{Mb} / \mathrm{s}$ and the maximum capacity is equal to $11 \mathrm{Mb} / \mathrm{s}$.

\begin{tabular}{|l|c|c|c|}
\hline $\begin{array}{l}\text { Network } \\
\text { Capacity }\end{array}$ & Minimum & Average & Maximum \\
\hline ETT & $9 \mathrm{Mb} / \mathrm{s}$ & $10 \mathrm{Mb} / \mathrm{s}$ & $11 \mathrm{Mb} / \mathrm{s}$ \\
\hline MIC & $10 \mathrm{Mb} / \mathrm{s}$ & $11 \mathrm{Mb} / \mathrm{s}$ & $12 \mathrm{Mb} / \mathrm{s}$ \\
\hline LAETT & $17 \mathrm{Mb} / \mathrm{s}$ & $18 \mathrm{Mb} / \mathrm{s}$ & $19 \mathrm{Mb} / \mathrm{s}$ \\
\hline
\end{tabular}

Table 4: Network Capacity

If we consider the LAETT metric, the average network capacity reaches $18 \mathrm{Mb} / \mathrm{s}$ (min: $17 \mathrm{Mb} / \mathrm{s}$ and max: $19 \mathrm{Mb} / \mathrm{s}$ ). This is nearly twice better than the one obtained with the ETT and MIC metrics.

The load distribution patterns remain similar to those of pure Internet traffic: congestion areas are still localized around the 4 IGWs. One could have expected a more natural balance of flows in the network (since the demand is better distributed).

To analyse what happens we took a closer look at which paths stay in the WMNs and which go through the Internet. Remember that Internet links have a negligible cost so there is a benefit to route intra-mesh traffic through IGWs across the Internet.

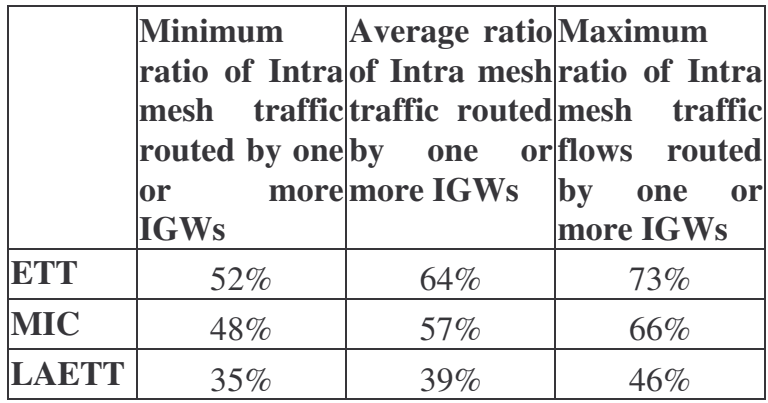

Table 5: Routing decision for intra-mesh traffic flows

Table 5 shows the ratio of intra-traffic flows that reach their destinations either via Internet or using an adjacent IGW MR. Using ETT this average ratio, on the 200 simulations, is equal to $64 \%$. Using MIC this 
average ratio is equal to $57 \%$. Finally, using the LAETT metric, the average ratio is reduced to $39 \%$.

In other words, with the LAETT metric, more of the intra-mesh traffic remains within the WMN, thus reducing the load at the IGWs, here again improving network capacity.

\section{Conclusion}

In this work we explored the benefits of introducing a load-dependent routing metric to increase WMN capacity and performance. We proposed a load aware isotonic routing scheme that uses weighted shortest path routing to balance the load across the network. The critical component of the scheme is a weight metric, called LAETT, that captures both traffic load and link quality.

To avoid congestion the scheme aims at maximizing the minimum remaining capacity of nodes in the network. This is achieved through the LAETT metric that is derived from the ETT metric to include estimates of the remaining capacity on the mesh nodes. The two main goals of the scheme are to provide a path which satisfies the bandwidth request of the flow and to leave room for future requests by balancing the load across the network. We compared the performances of LAETT to the two state-of-the art load independent metrics ETT and MIC and showed increased performance both in terms of network capacity and load distribution.

These results may motivate other studies that would aim to benefit from combining wireless characteristics and load estimates to achieve good utilization of the WMN capacity. Different air interfaces (contention based, CDMA or TDMA), different operating conditions (indoors or outdoors) or traffic types may lead to different solutions. Introducing load-dependent information in the routing scheme also needs careful system analysis to avoid possible instabilities. Some possible solutions are proposed in [14].

In our future work we plan to implement LAETT and experiment with it on a Mesh test bed with multiradio/multi-channel nodes and evaluate its performances under real conditions.

\section{Acknowledgements}

This work was partially funded by the European Commission Programme ICT-2008-215320 through the EU-MESH Project.

\section{References}

[1] Akyildiz, I., Wang, X., Wang, W., "Wireless mesh networks: a survey". Computer Networks, vol. 47, pp. $445-487$ (2005)

[2] Jun, J., Sichitiu, M.: The nominal capacity of wireless mesh. networks, IEEE Wireless Commun. Mag., vol. 10(5), pp. 8--14 (2003)

[3] De Couto, D., Aguayo, D., Bicket, J., Morris, R. : Highthroughput path metric for multi-hop wireless routing. In Mobicom'03 (2003)

[4] Y. Yang, J. Wang and R. Kravets, Load- balanced Routing For Mesh Networks ACM Mobile Computing and Communications Review (MC2R), 2006.

[5] R. Draves, J.Padhye, and B. Zill, "Routing in MultiRadio, Multi-Hop Wireless Mesh Networks," in Proc. of ACM MobiCom, 2004.

[6] M. Genetzakis and V. A. Siris, "A Contention-Aware Routing Metric for Multi-Rate Multi-Radio Mesh Networks", In Proc. of Fifth Annual IEEE Communications Society Conference on Sensor, Mesh and Ad Hoc Communications and Networks (SECON), San Francisco, June 2008.

[7] Allard, G., Jacquet, P.: Heuristics for bandwidth reservation in multihop wireless networks. In INRIA Research Report RR5075 (2004)

[8] Lakshmanan, S. Sundaresan, K. Sivakumar, R.: On multi-gateway association in wireless mesh networks, In Proceedings of WiMesh, IEEE Workshop on Wireless Mesh Networks (2006)

[9] H. Zhai and Y. Fang, "Impact of Routing Metrics on Path Capacity in Multi-rate and Multi-hop Wireless Ad Hoc Networks," in Proc. of IEEE Int'l Conference on Network Protocols (ICNP), 2006.

[10] J. L. Sobrinho, "Algebra and algorithms for QoS path computation and hop-by-hop routing in the Internet," in IEEE INFOCOM, 2001.

[11] Herve Aiache, Vania Conan, Laure Lebrun, Jeremie Leguay, Stephane Rousseau, Damien Thoumin. XIAN Automated Management and Nano-Protocol to Design Cross-Layer Metrics for Ad hoc Networking. IFIP Networking 2008. Singapore - May 2008.

[12] Gupta, P, Kumar, P.R., "The capacity of wireless networks", IEEE Trans. on Info Theory (2000)

[13] Jain, K., Padhye, J., Padmanabhan, V., Qiu, L., "The impact of interference on multi-hop wireless network performances". MOBICOM (2003)

[14] Chun-Yuan Chiu and Yu-Liang Kuo and Hsiao-Kuang $\mathrm{Wu}$ and Gen-Huey Chen, "Bandwidth constrained routing problem in multi-hop wireless networks", MSWiM '06: Proceedings of the 9th ACM international symposium on Modeling analysis and simulation of wireless and mobile systems, pages 365-369,2006

[15] IEEE. Standard 802.16-2004. Part16: Air interface for fixed broadband wireless access systems. October 2004. 